\title{
SAMPEX Measurements of Heavy Ions Trapped in the Magnetosphere
}

\author{
J. R. Cummings, A. C. Cummings, R. A. Mewaldt, \\ R. S. Selesnick, and E. C. Stone \\ California Institute of Technology, Pasadena CA 91125 \\ T. T. von Rosenvinge \\ NASA/Goddard Space Flight Center \\ Greenbelt, MD 20771 \\ J. B. Blake \\ The Aerospace Corporation \\ Los Angeles, CA 90009
}

\begin{abstract}
New observations of $>15 \mathrm{MeV} /$ nuc trapped heavy ions with $Z \geq 2$ have been made by the SAMPEX spacecraft in low polar orbit. The composition of these ions, which are located primarily around $\mathrm{L}=2$, is dominated by $\mathrm{He}, \mathrm{N}, \mathrm{O}$, and $\mathrm{Ne}$. The $\mathrm{N}, \mathrm{O}$, and $\mathrm{Ne}$ ions are apparently trapped "anomalous cosmic rays," while the origin of the trapped He flux is presently uncertain. These ions can affect the rate of singleevent upsets (SEUs) in spacecraft hardware.
\end{abstract}

\section{INTRODUCTION}

The Solar, Anomalous, and Magnetospheric Particle Explorer (SAMPEX) was launched July 3, 1992 into an $82^{\circ}$ inclination orbit with an apogee of $\sim 670 \mathrm{~km}$ and a perigee of $-520 \mathrm{~km}$ [1]. SAMPEX carries four energetic-particle instruments, including a Mass Spectrometer Telescope (MAST) designed to measure the elemental and isotopic composition of nuclei from $\mathrm{He}$ to $\mathrm{Ni}(\mathrm{Z}=2$ to 28 ) over the energy range from $\sim 15$ to $\sim 200 \mathrm{MeV} /$ nuc. Although MAST is designed primarily for studies of solar flare and cosmic-ray nuclei during its passage over the geomagnetic poles, trapped particles account for a significant fraction of the events with $Z \geq 6$ observed during the first few months of the mission. The vast majority of the ions with $Z \geq 6$ appear to be "anomalous cosmic rays" (ACRs) that have become trapped in the magnetosphere.

The interplanetary ACR component of the Galactic Cosmic Rays (GCRs) includes those elements (He, C, N, O, Ne, Ar, and perhaps $\mathrm{H}$ ) whose solar-minimum energy spectra have shown anomalous increases in flux above the quiet time GCR spectrum at energies $<50 \mathrm{MeV} /$ nuc (see, e.g., $[2,3]$ ). ACRs are thought to represent a sample of neutral interstellar particles that have drifted into the heliosphere, become ionized by the solar wind or UV radiation, and then accelerated to energies up to tens of $\mathrm{MeV} / \mathrm{nuc}$ [4]. This model predicts that ACRs should be singly ionized (for which there is recent evidence), in contrast to galactic cosmic rays, which are essentially fully stripped. Experimental evidence confirms that the ACRs are singly ionized [5]. Because ACRs are especially sensitive to solar modulation, they are detectable at $1 \mathrm{AU}$ only near solar minimum.

Soon after the discovery of ACRs, Blake and Friesen [6] suggested a mechanism for trapping ACR nuclei in the magnetosphere (see also Blake [7] and Schulz et al. [8]). T rapping can occur when a singly charged ion, with a rigidity somewhat above the geomagnetic cutoff, penetrates deeply into the magnetosphere and loses some or all of its remaining electrons in the upper atmosphere, such that its resulting rigidity is suddenly below the trapping limit. In 1991, Grigorov et al. [8] reported the first evidence for trapped ACRs. In that study, passive track detectors flown on COSMOS spacecraft during the 1985 to 1988 solar minimum observed $\sim 5$ to $30 \mathrm{MeV} /$ nuc ions with a composition, angular distribution, and temporal behavior consistent with the origin proposed by Blake and Friesen. However, the passive detectors on COSMOS could not measure the ions' spatial distribution.

In this paper, we report on a new study of the spatial distribution, composition, and energy spectrum of trapped heavy ions that began with the launch of SAMPEX in mid-1992. Initial results from this study have been reported by Cummings et al. $[10,11]$. We summarize here the current status of this work and discuss possible implications of these observations for the single-event-upset environment experienced by orbiting spacecraft.

\section{THE INSTRUMENT}

MAST is composed of an array of silicon solid-state detectors that determine the nuclear charge, mass, kinetic energy, and trajectory of particles that stop in the detector array (for a complete description, see [11]). The threshold for particle identification ranges from $14 \mathrm{MeV} /$ nuc for $\mathrm{C}$, to $16 \mathrm{MeV} / \mathrm{nuc}$ for $\mathrm{O}$, to $27 \mathrm{MeV} / \mathrm{nuc}$ for $\mathrm{Fe}$, with a more limited response to He from $~ 7.5$ to $-15 \mathrm{MeV} / \mathrm{nuc}$. The MAST telescope has a full-angle field of view of $\sim 101^{\circ}$ and a typical geometry factor of $-12.5 \mathrm{~cm}^{2} \mathrm{sr}$, oriented approximately toward local zenith over most of the SAMPEX orbit. The arrival time of incident nuclei can be measured to an accuracy of $\pm 1 \mathrm{~s}$. Because nuclei with $Z>2$ are given highest readout priority and because 
MAST is relatively insensitive to protons, it is capable of measuring heavy nuclei in the presence of a much greater flux of protons and/or electrons.

\section{OBSERVATIONS}

We describe below the essential features of trapped heavy nuclei observed by MAST over the period from $7 / 6 / 92$ to 2/7/93.

\section{A. Invariant Latitude Distributions}

Figure 1 shows the geographic distribution of oxygen nuclei with 16 to $200 \mathrm{MeV} /$ nuc observed by MAST during solar quiet times. Over the magnetic poles, MAST can detect galactic cosmic rays mixed with a solar-cycle-dependent contribution from -15 to $-40 \mathrm{MeV} /$ nuc ACR nuclei. These highlatitude particles are present at all longitudes. Also evident in Figure 1 is a trapped population concentrated in the region south-east of the South Atlantic Anomaly (SAA), which extends to energies $>50 \mathrm{MeV} /$ nuc. (The particles appear in the SAA region because the relatively low magnetic field in this region allows the ions to dip down to the SAMPEX altitude there; the mirror-point altitude follows a locus of constant magnetic field.) Tylka [13] has produced Monte Carlo models of trapped anomalous cosmic rays that bear an excellent resemblance to this plot. Figure 2 shows invariant latitude distributions for $\mathrm{He}$ and $\mathrm{O}$, two of the species observed in this region. Note that the distribution of $O$ is sharply peaked at $\Lambda$ $=45^{\circ}$, corresponding to $\mathrm{L}=2$. The intensity of energetic oxygen nuclei in this region exceeds that over any portion of the SAMPEX orbit, except for those rare periods when very large solar-particle events are observed over the poles $(\Lambda>$ $\left.65^{\circ}\right)$.

The location of the trapped oxygen fluxes is somewhat energy dependent, with higher-energy oxygen trapped on lower L shells. At a given energy/nucleon, $E$, more than $\sim 90 \%$ of the oxygen events are within a region of $L$ bounded by

$$
L(E)=(2.0 \pm 0.3)(E / 20 \mathrm{MeV} / \text { nuc })^{-0.25} \text {. }
$$

We also observe that the trapped $\mathrm{N}$ and $\mathrm{Ne}$ fluxes have a similar L-shell distribution.

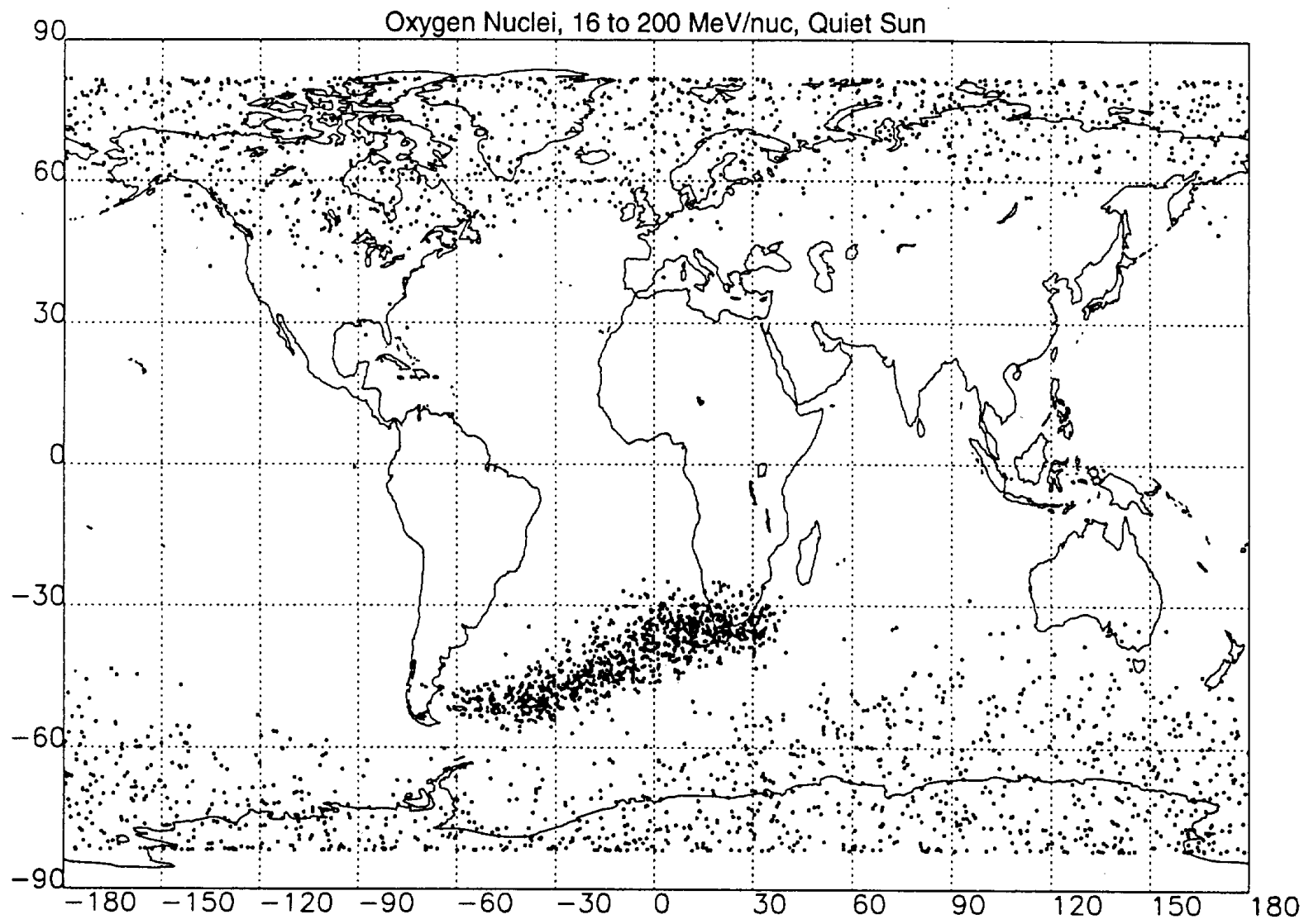

Fig. 1. Geographic distribution of quiet-time oxygen nuclei with 16 to $200 \mathrm{MeV} /$ nuc observed by MAST during the period from $7 / 6 / 92$ to $2 / 6 / 93$. For comparison, the SAA is centered approximately at $\sim 30^{\circ}$ South Latitude and $-315^{\circ}$ degrees Longitude. 

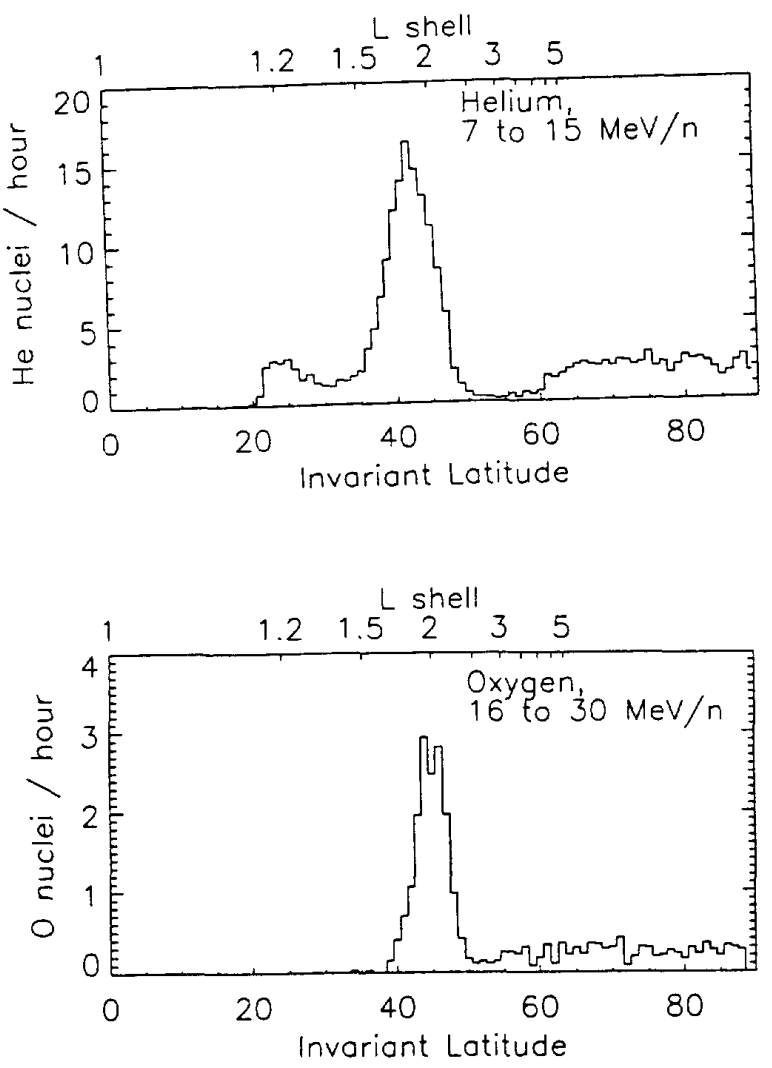

Fig. 2. Invariant latitude distribution of quiet-time $\mathrm{He}$ and $\mathrm{O}$ observed by MAST.

The trapped He observed by MAST has a somewhat different latitude distribution than that of heavier ions, with a peak at $L=1.8$, approximately independent of energy, and a somewhat smaller intensity peaked at $L=1.2$, in the region of the SAA. Note that the MAST instrument is sensitive to He only over the energy range from $\sim 7.5$ to $\sim 15 \mathrm{MeV} / \mathrm{nuc}$, much lower in energy than its response to heavier nuclei [11].

\section{B. Composition}

As Figure 3 indicates, the composition of trapped nuclei with $\mathrm{Z} \geq 6$ that is observed at $\mathrm{L} \approx 2$ is dominated by $\mathrm{N}, \mathrm{O}$, and $\mathrm{Ne}$ (see also [14]). In addition, one event each have been observed of $B$ and $C$ (along with a single event having $Z=23$ ). It should be pointed out that the composition is somewhat energy dependent (see below), which may explain why the COSMOS observations at somewhat lower energy [9] include less $\mathrm{N}$ and somewhat more $\mathrm{Ne}$.

\section{Pitch-angle Distributions}

As the orbit of SAMPEX precesses, the angle of the MAST telescope with respect to the local magnetic field in the $L=2$ region varies with a period of 3 months. Figure 4 shows that the particles at $\mathrm{L}=2$ are observed primarily when the MAST telescope is viewing nearly perpendicular to the local magnetic-field line, implying that these are indeed trapped particles with pitch angles of $\sim 90^{\circ}$.

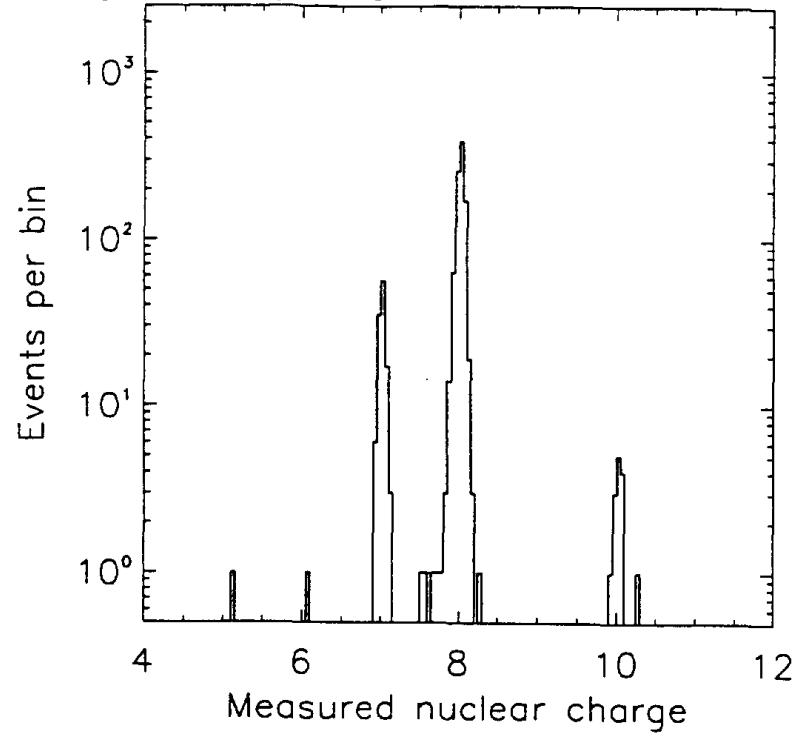

Fig. 3. Observed composition of trapped nuclei with $\mathrm{Z}>4$ at $\mathrm{L}=$ $2.05 \pm 0.60$, including all days from $7 / 6 / 92$ to $2 / 7 / 93$. Detection thresholds in MAST range from $\sim 14 \mathrm{MeV} /$ nuc for $\mathrm{C}$, to -16 $\mathrm{MeV} /$ nuc for $\mathrm{O}$, to $-18 \mathrm{MeV} / \mathrm{nuc}$ for $\mathrm{Ne}$. In addition, one event with $Z=23$ was observed.

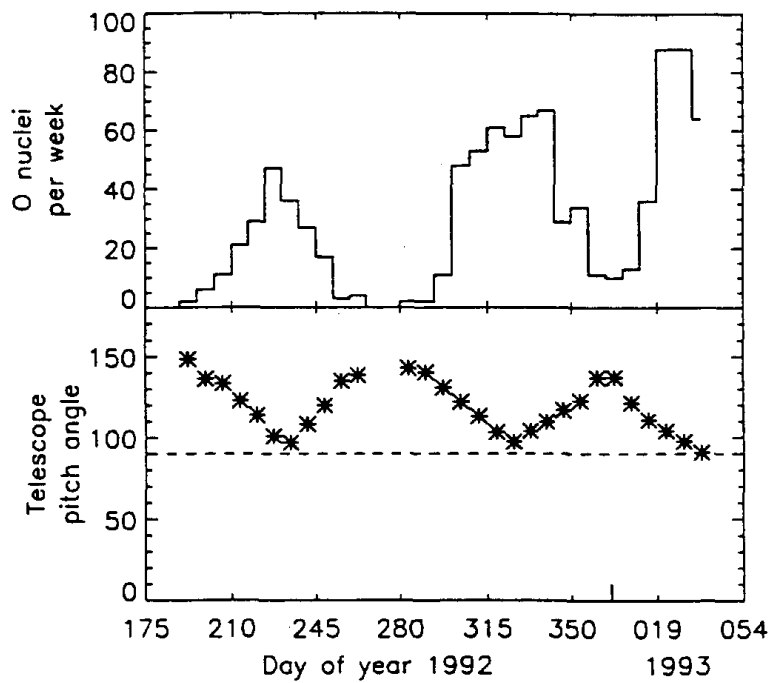

Fig. 4. Top Panel: Rate at which MAST detects $O$ nuclei in the region Southeast of the SAA, delimited here by $L=2.05 \pm 0.60$ and restricted to the Southern Hemisphere. Bottom Panel: Average angle of the MAST telescope axis to the local magnetic field (calculated from the 1990 IGRF, updated with secular variations) for events included in the top panel. This angle evolves with a -3-month period as the SAMPEX orbit precesses. The MAST telescope has a full opening angle of $\sim 101^{\circ}$. Similar results are obtained for $\mathrm{He}, \mathrm{N}$, and $\mathrm{Ne}$. 


\section{Time Variations}

Grigorov et al. [9] found that the intensity of the trapped oxygen fluxes measured over the years from 1985 to 1988 varied by a factor of $\sim 10$, in good correlation with the interplanetary intensity of ACR oxygen measured by IMP-8. Over the course of the SAMPEX mission, the ACR fluxes at $L=2$ have increased by a factor of -2 (see Figure 4 ), consistent with the interplanetary ACR flux measured by MAST as it passes over the geomagnetic poles [15]. Since the interplanetary ACR fluxes are the source of these trapped nuclei, we would expect the intensity of the trapped fluxes to continue building up as solar minimum approaches over the next few years. During the 1987 solar minimum, Bobrovska et al. [14] concluded that the flux of trapped ACRs at $\sim 200$ to $400 \mathrm{~km}$ was $\sim 500$ times greater than the interplanetary intensity

\section{ORIGIN OF THE TRAPPED HEAVY IONS}

Both Grigorov et al. [9] and Cummings et al. [9,10] argued that on the basis of the observed composition, angular distribution, and time variations, the trapped heavy nuclei observed first by COSMOS satellites and now by SAMPEX must represent trapped anomalous cosmic rays, presumably trapped by the mechanism proposed by Blake and Friesen [6]. Although the observations to date are generally consistent with that model, the SAMPEX observations now show that there are differences in detail. In particular, we find that the trapped ACRs are located closer to Earth than was predicted.

SAMPEX observations of interplanetary cosmic rays [15] verify that there is an interplanetary source of anomalous $\mathbf{N}$, $\mathrm{O}$, and $\mathrm{Ne}$ in late 1992 with sufficient energy to exceed the geomagnetic cutoff for particles arriving at $L=2$ from the west. As discussed by Cummings et al. [11], the appearance of trapped $\mathrm{He}$ at $\mathrm{L}=2$ is somewhat surprising because $\mathrm{He}$ is not expected to be trapped by the Blake and Friesen mechanism [6]. To reach $\mathrm{L}=1.8$ where it is observed, (see Figure 2) singly ionized He arriving from the west would require $>300 \mathrm{MeV} / \mathrm{nuc}$, well above the maximum energy to which it is observed in interplanetary space. If the trapped $\mathrm{He}$ is of ACR origin, there must be some other mechanism by which it is trapped. On the other hand, Adams [16] and Blake [17] have suggested that the observed $\mathrm{He}$ ions might be a remnant of a presumably solar particle population injected by the unusually strong and temporally narrow shock that struck the Earth's magnetosphere at 03:42 UT on 24 March 1991. The results of the injection were observed by CRRES [ 18,19,20]. In this case, the $\mathrm{He}$ intensity should decrease with time, while the intensity of trapped ACR species is expected to increase with time as the interplanetary intensity of the ACRs increases during the approach to solar minimum. Preliminary results indicate that the trapped He flux is decreasing with time.

\section{RADIATION EFFECTS OF TRAPPED ACRS}

The potential of trapped ACRs to cause SEUs in spacecraft hardware will of course depend on the spacecraft orbit and the degree to which sensitive electronics are shielded. We consider here only trapped ACR oxygen and limit our attention to the SAMPEX orbit. In Figure 5, we show the quiet-time energy spectrum of oxygen observed by MAST, averaged over the entire orbit, including galactic cosmic rays, interplanetary anomalous cosmic rays, and trapped anomalous cosmic rays. Since MAST is oriented perpendicularly to the local magnetic field at $L-2$ only $1 / 3$ of the time, an omnidirectional detector would show a greater countrate. It is clear that trapped and interplanetary ACRs can contribute a significant fraction of the linear energy transfer (LET) distribution due to GCRs, although they are rather easily shielded against because of their low energies. However, magnetospheric processes such as radial diffusion may increase the energy of the geomagnetically trapped GCRs. The temporal behavior of the trapped GCRs needs to be carefully followed over the next several years by SAMPEX, with special emphasis upon the effects of major geomagnetic storms upon the trapped ions.

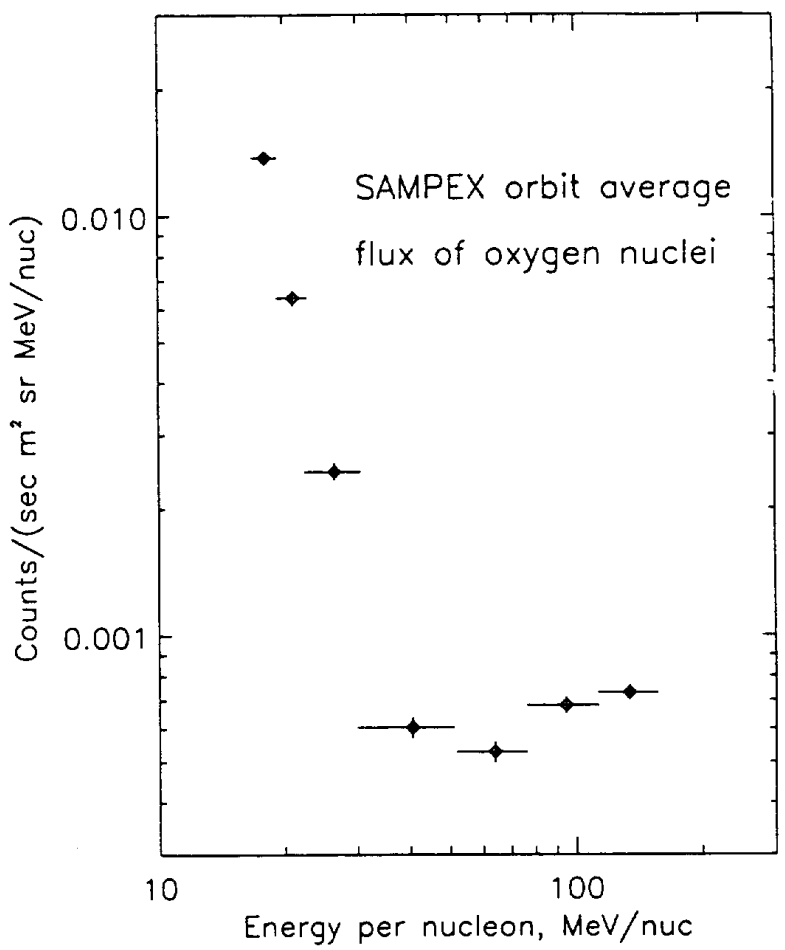

Fig. 5. Oxygen spectra averaged over the SAMPEX orbit during solar quiet times from 92:187 to 93:038. All event totals have been divided by the live time of the instrument, summed over the MAST orbit. Approximately $75 \%$ of the events in the rising portion of the spectrum are trapped ACRs. 
It also should be noted that in other orbits that spend more time around $L=2$, i.e., lower inclination orbits, the integrated trapped ACR exposure could be an order of magnitude or more larger. In addition, it is expected, based upon previous solar cycles, that the trapped ACR intensity will increase by up to a factor of $\sim 10$ over the next few years. SAMPEX measurements over the next few years will, therefore, be important in order to monitor the magnitude of SEU hazard that these trapped heavy nuclei may present to orbiting spacecraft. The energies of the trapped anomalous ions are relatively low. Shielding of the order of $100 \mathrm{mil}$ of aluminum is adequate to protect vulnerable electronic components, cf. Adams, Jr. and Partridge [21].

\section{ACKNOWLEDGMENTS}

This work was supported by NASA under contract NAS530704 and grant NAGW-1919 at the California Institute of Technology and under NASA Cooperative Agreement 26979B at The Aerospace Corporation.

\section{REFERENCES}

[1] D. N. Baker, G. M. Mason, O. Figueroa, G. Colon, J. G Watzin, R. M. Aleman, "An overview of the Solar, Anomalous, and Magnetospheric Particle Explorer (SAMPEX) Mission," IEEE Trans. on Geoscience and Remote Sensing, 31, p. 531, 1993.

[2] W. R. Webber, "Composition of anomalous cosmic rays, in Cosmic Abundances of Matter," AIP Conf. Proceedings 183 C. J. Waddington, ed., p. 100, 1989.

[3] A. C. Cummings, and E. C. Stone, "Elemental composition of the very local interstellar medium as deduced from observations of anomalous cosmic rays," Proc. 21st Internat. Cosmic Ray Conf. (Adelaide) vol. 6, p. 202, 1990.

[4] L. A. Fisk, B. Kozlovsky, and R. Ramaty, "An interpretation of the observed oxygen and nitrogen enhancements in low-energy cosmic rays," Ap. J. (Ltrs.), vol. 190, p. 35 , 1974.

[5] J. H. Adams, Jr., M. Garcia-Muñoz, N. L. Gregorov, B. Klecker, M. A. Kondratyena, G. M. Mason, R. E. ;McGuire, R. A. Meuraldt, M. I. Panaysuk, Ch. A. Tretyakona, A. J. Tylka, and D. A. Zhuravlev, Ap. J. Lett., 375, p. 145, 1991.

[6] J. B. Blake, and L. M. Friesen, "A technique to determine the charge state of the anomalous low energy cosmic rays," Proc.1Sth Internat. Cosmic Ray Conf. (Plovdiv) vol. 2, p. $341,1977$.
[7] J. B. Blake, "Geomagnetically trapped heavy ions from anomalous cosmic rays," Proc. 21st Internat. Cosmic Ray Conf. (Adelaide) vol. 7, p. 30, 1990.

[8] M. Schulz, J.B. Blake, and M. McNab, "Dynamical model for the distribution of geomagnetically trapped anomalouscomponent ions," J. Geophys. Res. , in press, 1993.

[9] N. L. Grigorov, M. A. Kondratyeva, M. I. Panasyuk, Ch. A. Tretyakova, J. H. Adams, Jr., J. B. Blake, M. Schulz, R. A. Mewaldt, and A. J. Tylka, "Evidence for anomalous cosmic ray oxygen ions in the inner magnetosphere," Geo. Res. (Ltrs.) vol. 18, p. 1959, 1991.

[10] J. R. Cummings, A. C. Cummings, R. A. Mewaldt, R. S. Selesnick, E. C. Stone, and T. T. von Rosenvinge, "New evidence for anomalous cosmic rays trapped in the magnetosphere," Proc. 23rd Internat. Cosmic Ray Conf. (Calgary) vol. 3, p. 4281993 ..

[11] ibid., "New evidence for geomagnetically trapped anomalous cosmic rays," submitted to Geophys. Res. Lett., June 1993.

[12] W. R. Cook, A. C. Cummings, J. R. Cummings, T. L. Garrard, B. Kecman, R. A. Mewaldt, R. S. Selesnick, E. C. Stone, and T. T. von Rosenvinge, "MAST: A Mass Spectrometer Telescope for studies of the isotopic composition of solar, anomalous, and galactic cosmic ray nuclei," IEEE Trans. on Geoscience and Remote Sensing, 31, p. 557,1993

[13] A. Tylka, "Spectra and geographical distribution of geomagnetically trapped anomalous cosmic rays," Proc. 23rd Intemat. Cosmic Ray Conf. (Calgary) 1993.

[14] V. V. Bobrovskaya, N.L. Grigorov, M.A. Kondratyeva, M.I. Panasyuk, Ch. A. Tretyakova, D.A. Zhuravlev, J.H. Adams, Jr. and A.J. Tylka, "Cosmos observations of anomalous cosmic ray $\mathrm{N}$ and $\mathrm{Ne}$ in the inner magnetosphere," Proc. 23rd Internat. Cosmic Ray Conf. (Calgary) 1993.

[15] R. A. Mewaldt, A. C. Cummings, J. R. Cummings, R. A. Mewaldt, E. C. Stone, and T. T. von Rosenvinge, "The return of the anomalous cosmic ray component to $1 \mathrm{AU}$ in 1992," submitted to the 23rd Internat. Cosmic Ray Conf. (Calgary) vol. 3, p. 432, 1993.

[16] J. H. Adams, Jr., private communication, 1993.

[17] J. B. Blake, private communication, 1993.

[18] J. B. Blake, W. A. Kolasinski, R. W. Fillius, and E. G. Mullen, "Injection of electrons and protons with energies of tens of MeV into L < 3 on 24 March 1991," Geophys. Res. Lett. vol. 19, p. 821, 1992.

[19] J. B. Blake, M. S. Gussenhoven, E. G. Mullen, and R. W. Fillius, "Identification of an Unexpected Space Radiation Hazard, IEEE Trans. on Nuc. Sci. vol. 39, p. 1761, 1992.

[20] A. L. Vampola, and A. Korth, "Electron Drift Echoes in the Inner Magnetosphere, Geophys. Res. Lett., vol. 19, p. 625, 1992.

[21] J. H. Adams, Jr. and K. Partridge, "Do Trapped Heavy Ions Cause Soft Upsets on Spacecraft?" NRL Memorandum Report 4846, 12 October 1982. 